\title{
Vom Verfassungskonvent zurück zur ,Methode Monnet'? Die Entstehung der ,Road map‘ zum EU-Reformvertrag unter deutscher Ratspräsidentschaft
}

\author{
Wolfgang Wessels und Anne Faber*
}

In den vergangenen Jahrzehnten hat die Europäische Gemeinschaft/Europäische Union, wie in der integration der letzten 30 Jahre umfassend dokumentiert und analysiert, ${ }^{1}$ unterschiedliche Verfahren bei der (quasi-)konstitutionellen Gestaltung des Systems der Europäischen Union erprobt. Zu beobachten sind vielfältige Aktivitäten und Bemühungen wie (in)formelle Treffen der europäischen Staats- und Regierungschefs, Regierungskonferenzen und - in diesem Jahrzehnt - auch zwei ,Konvente', in deren Rahmen Entwürfe für wesentliche Bestandteile eines neuen Grundlagenvertrags erarbeitet wurden. Zu Beginn dieses Jahrzehnts schien sich dabei ein Paradigmenwechsel abzuzeichnen, der die bis dahin klassischen Arten der Systemgestaltung, die ,Methode Monnet ${ }^{2}{ }^{2}$ sowie Treffen des Europäischen Rates mit anschließenden Regierungskonferenzen, durch eine neue Vorgehensweise, die Konventsmethode, zu ergänzen wenn nicht sogar zu ersetzen versuchte. ${ }^{3}$

Nach dem Scheitern des Vertrags über eine Verfassung für Europa in den Referenden in den Niederlanden und in Frankreich war es jedoch erneut der Europäische Rat, der - im Anschluss an eine ausgedehnte Reflexionsphase ${ }^{4}-$ mit der Verabschiedung eines Mandats für eine Regierungskonferenz am 21./22. Juni 2007 einen weiteren Meilenstein in der Geschichte der europäischen Integrationskonstruktion setzte. In 35 Stunden teilweise dramatischer und zugespitzter Verhandlungen legten die Staats- und Regierungschefs der 27 Mitgliedstaaten unter dem Vorsitz der deutschen Bundeskanzlerin Angela Merkel nicht nur einen Fahrplan (eine ,Road map') für die kommenden Monate fest, ${ }^{5}$ sondern verabschiedeten auch zugleich in einem „Mandat für die Regierungskonferenz" ${ }^{\circ 6}$ wesentliche Inhalte für konkrete Vertragsreformen. Die portugiesische Präsidentschaft hat auf dieser Grundlage ei-

* Prof. Dr. Wolfgang Wessels, Jean Monnet Lehrstuhl, Forschungsinstitut für Politische Wissenschaft und Europäische Fragen, Universität zu Köln.

Dr. Anne Faber, Wissenschaftliche Mitarbeiterin, Jean Monnet Lehrstuhl Prof. Dr. Wolfgang Wessels, Forschungsinstitut für Politische Wissenschaft und Europäische Fragen, Universität zu Köln.

Die Autoren danken Niklas Helwig und Christina Zuber für die vielfältige Zuarbeit zu diesem Beitrag.

1 Übersicht im Online-Archiv der integration, abrufbar unter: http://www.iep-berlin.de/index.php?id=suche_db (letzter Zugriff: 21.09.2007).

2 Der im folgenden Text verwendete Begriff der ,Methode“ wird dabei synonym zu den Begriffen ,Verfahren“ und ,Strategie' verwendet und bezieht sich im Unterschied zum Begriff der ,Politikgestaltung' auf Akteursstrategien im Hinblick auf die ,Systemgestaltung' der Europäischen Union.

3 Vgl. Joschka Fischer: Vom Staatenverbund zur Föderation - Gedanken über die Finalität der Europäischen Integration, Rede vom 12.5.2000 an der Humboldt-Universität Berlin, abgedruckt in: integration 3/2000, S. 149 156; Wolfgang Wessels: Der Konvent: Modelle für eine innovative Integrationsmethode, in: integration 2/2002, S. 83-98; Brigid Laffan: Der schwierige Weg zur Europäischen Verfassung: Von der Humboldt-Rede Außenminister Fischers bis zum Abschluss der Regierungskonferenz, in: Mathias Jopp/Saskia Matl (Hrsg.): Der Vertrag über eine Verfassung für Europa. Analysen zur Konstitutionalisierung der EU, Baden-Baden 2005, S. 473-492.

4 Vgl. Daniel Göler/Hartmut Marhold: Die Zukunft der Verfassung - Überlegungen zum Beginn der Reflexionsphase, in: integration 4/2005, S. 332-335.

5 Vgl. Europäischer Rat (Brüssel): Schlussfolgerungen des Vorsitzes, 21./22. Juni 2007, Ziffer 10 und 11, abrufbar unter: http://www.consilium.europa.eu/ueDocs/cms_Data/docs/pressData/de/ec/94935.pdf (letzter Zugriff: 14.08.2007).

6 Europäischer Rat (Brüssel): Mandat für die Regierungskonferenz 2007, 23.06.2007, abrufbar unter: http://register.consilium.europa.eu/pdf/de/07/st11/st11218.de07.pdf (letzter Zugriff: 14.08.2007). 
nen Entwurf für einen Reformvertrag vorgelegt, ${ }^{7}$ den die Regierungskonferenz im zweiten Halbjahr 2007 zur Unterschriftsreife führen soll. Daneben ist in den letzten Wochen vom französischen Staatspräsidenten Nicolas Sarkozy und unterstützt von Bundeskanzlerin Angela Merkel der Vorschlag gemacht worden, einen ,Rat der Weisen` einzuberufen, der Eckpunkte für die weitere Ausgestaltung der Europäischen Union und ihre geografische finalité erarbeiten soll. ${ }^{8}$ Auf nationaler wie auf europäischer Ebene stößt dieser Vorschlag jedoch auf Skepsis. So kritisiert der Vorsitzende des Verfassungsausschusses im Europäischen Parlament, Jo Leinen (SPD): „Dies ist ein Rückfall in die Methoden der 60er und 70er Jahre. Statt eines ,Rat der Weisen “ [sic!] muss vielmehr ein neuer Konvent einberufen werden“.9

Die erneute Debatte zeigt exemplarisch die fortbestehenden Differenzen bei der Suche nach dem ,Königsweg ' zur zukünftigen Systemgestaltung. Der folgende Beitrag stellt Regierungskonferenzen, die Konventsmethode sowie die ,Methode Monnet' als die drei relevanten Verfahren bei der Weiterentwicklung der Europäischen Union vor und beleuchtet ihre jeweiligen Charakteristika, Stärken und Schwächen aus politischer und integrationstheoretischer Perspektive. Von diesen drei Verfahrensoptionen ausgehend wird die Vorgehensweise der deutschen Ratspräsidentschaft unter Angela Merkel analysiert. Dabei wird argumentiert, dass unter der deutschen Ratspräsidentschaft im ersten Halbjahr 2007 eine Renaissance der ,Methode Monnet" festzustellen ist, die von der Bundesregierung weiterentwickelt und ausgebaut wurde. Das Zustandekommen der ,Road map “ unter der Präsidentschaft Merkel ist so in einer Gesamtperspektive als Ergebnis eines Zusammenspiels der Konventsmethode, der , Methode Monnet' und der Verhandlungen auf der Ebene der Staats- und Regierungschefs im Europäischen Rat zu diskutieren. Vor diesem Hintergrund erscheint eine Debatte über die Frage, welche die ,beste' Methode zur Erarbeitung von Vorschlägen für die Zukunft der Europäischen Union ist, wenig konstruktiv. Der Blick sollte sich vielmehr auf die jeweiligen Stärken und Schwächen der drei Methoden und ihren optimalen ,Mix ‘ als einander ergänzende Verfahren bei der Weiterentwicklung der Union richten.

\section{Regierungskonferenzen als Institution}

Solange von den vertraglichen Entwicklungen der letzten Jahrzehnte als Basis für jede weitere Systementwicklung ausgegangen, das heißt kein konstitutioneller Neuanfang geplant wird, stellen Regierungskonferenzen nach Artikel 48 EUV das Verfahren zur Änderung der relevanten Verträge dar. In diesem Sinne sind Regierungskonferenzen nicht eine von mehreren verschiedenen ,Methoden“ der Systementwicklung, sondern die im juristischen Sinne einzig zulässige und reguläre Institution zur Änderung der Verträge. Regierungskonferenzen repräsentieren damit die vertraglich fixierte Form, in der die Mitgliedstaaten das System der Europäischen Union immer wieder in quasi-konstitutioneller Form gestaltet und Kompetenzzuordnungen vorgenommen haben. ${ }^{10}$ Seit Beginn der Integrationskonstruktion verlaufen Re-

7 Entwurf eines Vertrags zur Änderung des Vertrags über die Europäische Union und des Vertrags zur Gründung der Europäischen Gemeinschaft, Brüssel 23. Juni 2007, abrufbar unter: http://www.consilium.europa.eu/ igcpdf/de/07/cg00/cg00001.de07.pdf (letzter Zugriff: 14.08.07).

8 Vgl. http://www.bundesregierung.de/nn_774/Content/DE/Mitschrift/Pressekonferenzen/2007/09/2007-09-10pk-merkel-sarkozy.html (letzter Zugriff: 21.09.2007).

9 http://www.spd-europa.de/news-anzeige/news/jo-leinen-neuer-konvent-statt-rat-der-weisen/29/neste/2.html (letzter Zugriff: 21.09.2007).

10 Zur Diskussion um den Begriff ,konstitutionell` siehe Stephan Hobe: Quo vadis Europäische Verfassung? Zwischen Vertrag und Verfassung - wie verfasst ist Europa? Eine rechtswissenschaftliche Betrachtung, in: Sozialer Fortschritt 5/2007, S. 113-146; Wolfgang Wessels: Die Europapolitik in der wissenschaftlichen Debatte, in: Werner Weidenfeld/Wolfgang Wessels (Hrsg.): Jahrbuch der Europäischen Integration 2006, Baden-Baden 2007, S. 27-38. 
gierungskonferenzen üblicherweise in drei ineinander übergehenden Phasen: der Initiativ-, der Verhandlungs- sowie der Ratifizierungsphase (vgl. Schaubild 1).

\section{Schaubild 1: Dreiphasenmuster von Regierungskonferenzen}

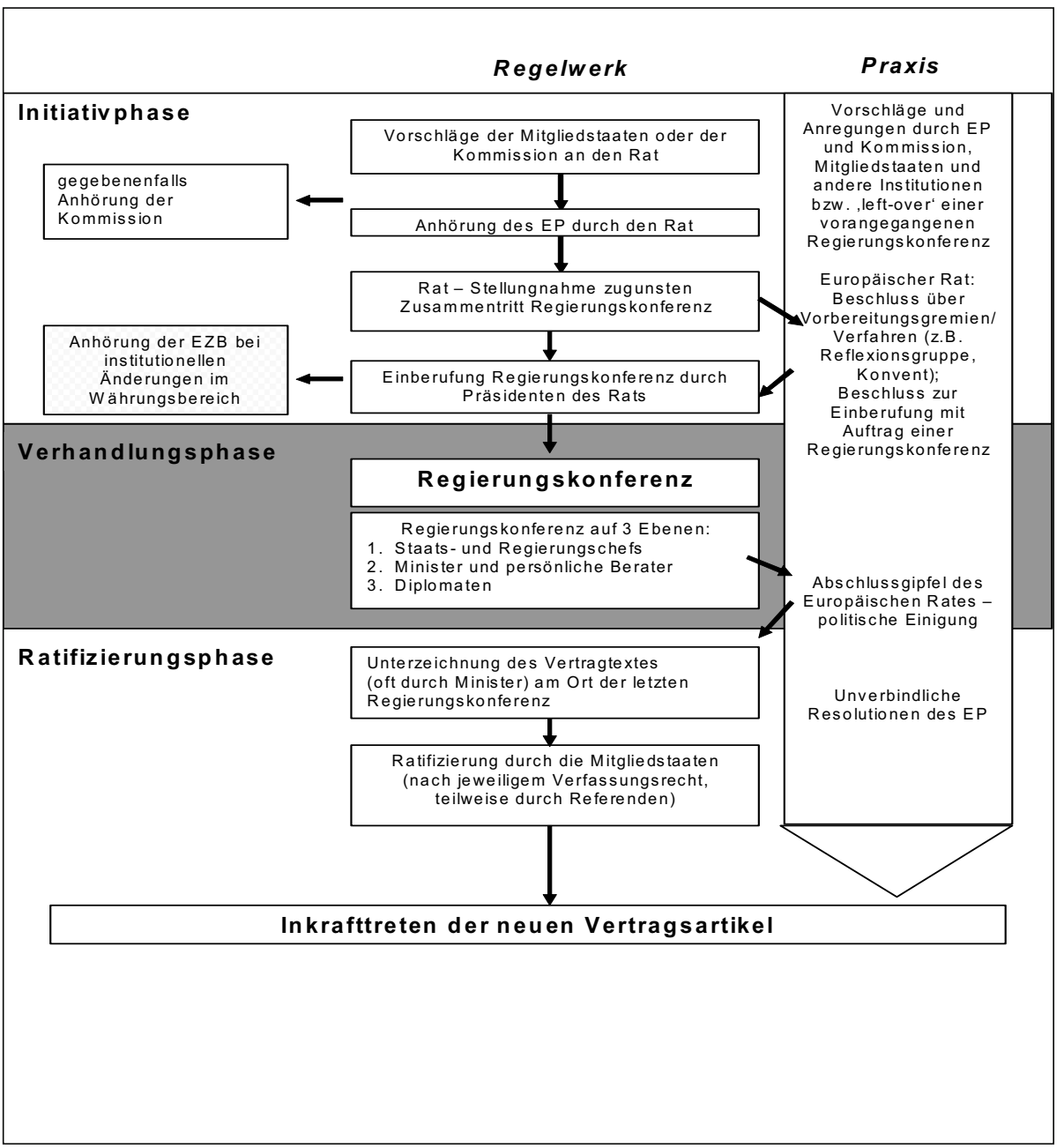

Quelle: Wolfgang Wessels: Das politische System der Europäischen Union, Wiesbaden (im Erscheinen)

Insbesondere die Initiativphase ist dabei von den Regierungen der Mitgliedstaaten sehr unterschiedlich gestaltet worden. ${ }^{11}$ So ist von einem (vom französischen Staatspräsidenten Sarkozy nun erneut geforderten) ,Rat der Weisen“ bis hin zu Konventen mit unterschied-

11 Vgl. die Analysen und Berichte aus der Zeitschrift integration; stellvertretend für viele: Daniel Göler/Mathias Jopp: Kann Europa gelingen? Vorhaben und Chancen der deutschen Ratspräsidentschaft, in: integration 1/ 2007, S. 3-24; Kai Oppermann: Die britische Ratspräsidentschaft 2005: Zwischen europäischen Erwartungen und innenpolitischen Restriktionen, in: integration 1/2006, S. 23-37. 
lichsten Formen und Instrumenten bei der Vorbereitung von Regierungskonferenzen experimentiert worden. Dazu zählen unter anderem der Tindemans-Bericht zur Europäischen Union, ${ }^{12}$ der Dooge-Ausschuss zur Vorbereitung der Einheitlichen Europäischen Akte, ${ }^{13}$ der Delors-Ausschuss für die Währungsunion sowie eine Reflexionsgruppe persönlicher Beauftragter zur Vorbereitung der Regierungskonferenz, die zum Amsterdamer Vertrag führte. ${ }^{14}$ Unter einem ,Rat der Weisen“ ist dabei klassischerweise eine Gruppe von drei oder mehr ,Weisen', oftmals ehemaligen Staats- und Regierungschefs, zu verstehen, wie zum Beispiel Jean-Luc Dehaene, David Simon und Richard von Weizsäcker und ihr Bericht an die Europäische Kommission über die institutionellen Auswirkungen der Erweiterung vom Oktober 1999. ${ }^{15}$ Der vom Europäischen Rat in Laeken 2001 einberufene Europäische Konvent zur Zukunft Europas kann ebenfalls als eine - wenn auch besondere - Variante dieser Initiativphase verstanden werden. Auch der Auftrag des Europäischen Rates an die Bundesregierung im Juni 2006, in ihrer Präsidentschaft einen ,Fahrplan' für die weitere Behandlung des Verfassungsvertrags vorzulegen, kann als eine spezifische Form der Vorbereitung gesehen werden. Mit diesen informellen, vorbereitenden Verfahren des ,Agenda-settings' sollen bereits im Vorfeld von Regierungskonferenzen die Erfolgsaussichten von Initiativen und Reformvorhaben getestet werden. In der Regel definiert dann der Europäische Rat bei der Einberufung der Regierungskonferenz den Spielraum, in dem sich die Verhandlungen bei der eigentlichen Regierungskonferenz bewegen können beziehungsweise sollten.

Die eigentlichen Regierungskonferenzen als zweite Phase haben sich zu Institutionen eigener Art entwickelt, die den nationalen Regierungen und anderen beteiligten Akteuren zumindest indirekt in der Praxis erprobte Muster für die Verfahren vorgeben. ${ }^{16}$ Sie können damit sogar als eine Art ,Meta-Institution“ der Systemgestaltung verstanden werden, die das Regelwerk für die Institutionen im engeren Sinne beschließt. ${ }^{17}$ In der Verhandlungsphase findet in einem Prozess des, intergovernmental bargaining ' der Austausch und Abgleich nationaler Interessen und Entwürfe statt. Vertragsänderungen beschließt der Rat dabei einstimmig und in der Regel nachdem der Europäische Rat auf einem Abschlussgipfel in einer ,Nacht der langen Messer` einen Konsens über die letzten strittigen Punkte erzielt hat.

Die Vertragsänderungen treten dann in Kraft, wenn jeder Mitgliedstaat, das heißt in den meisten Fällen die nationalen Parlamente, diese nach den jeweiligen verfassungsrechtlichen Verfahren ratifiziert hat. In einigen Mitgliedstaaten (zum Beispiel Irland) sind im Regelfall Referenden vorgeschrieben, in anderen ist die Befragung der Bevölkerung fakultativ. Dem Europäischen Parlament spricht der Vertrag - im Unterschied zum Beitrittsverfahren - kein Zustimmungsrecht zu. ${ }^{18}$ Die Abgeordneten des Europäischen Parlaments sind damit vom ei-

12 Vgl. Wolfgang Wessels: Die Integrationsstrategie des Tindemans-Berichts, in: Heinrich Schneider/Wolfgang Wessels (Hrsg.): Auf dem Weg zur Europäischen Union? Diskussionsbeiträge zum Tindemans-Bericht, Bonn 1977, S. 217-238.

13 Vgl. zum „Ad-hoc-Ausschuss für institutionelle Fragen“ („Dooge-Ausschuss“) Rudolf Hrbek: Welches Europa? Zum Zwischenbericht des Ad-hoc-Ausschusses für institutionelle Fragen (Dooge - Committee), in: integration 1/1985, S. 3-10.

14 Vgl. zur „Reflexionsgruppe“ Wolfgang Wessels: Weder Vision noch Verhandlungspaket - der Bericht der Reflexionsgruppe im integrationspolitischen Trend, in: integration 1/1996, S. 14-24.

15 Abrufbar unter: http://www.internationalepolitik.de/archiv/jahrgang1999/november99/bericht-an-die-europaische-kommission-uber-die-institutionellen-auswirkungen-der-erweiterung-von-jean-luc-dehaene--david-simonund-richard-von-weizsacker--vorgelegt-am-18--oktober-1999-in-brussel.html (letzter Zugriff: 21.09.2007).

16 Vgl. Thomas Christiansen: The role of supranational actors in EU treaty reform, in: Journal of European Public Policy 1/2002, S. 33-53, hier S. 34.

17 Vgl. Thomas Christiansen/Gerda Falkner/Knud Erik Jørgensen: Theorising EU Treaty Reform: Beyond Diplomacy and Bargaining, in: Journal of European Public Policy 1/2002, S. 12-32.

18 Bei den letzten Regierungskonferenzen nahmen zwei Abgeordnete des Europäischen Parlaments als Beobachter teil. 
gentlichen Ratifizierungsprozess ausgeschlossen und besitzen so auch keine Möglichkeit, über die Ausweitung ihrer eigenen Kompetenzen mit zu beschließen. Bei dieser Form der Systemgestaltung sehen die Vertragsbestimmungen vor, dass das Europäische Parlament ausschließlich bei der Einberufung von Regierungskonferenzen ,,angehört“ wird. Auch wenn die formalen Kompetenzen des Europäischen Parlaments im Rahmen von Regierungskonferenzen schwach ausgeprägt sind, kann davon ausgegangen werden, dass die Resolutionen und Debatten des Europäischen Parlaments indirekt und informell in Form einer präkonstitutionellen Normengenese nachhaltigen Einfluss auf die Verhandlungen ausüben.

Regierungskonferenzen stellen damit - zumindest auf den ersten Blick - das klassische intergouvernementale Verfahren zur Systementwicklung dar, das aus Perspektive des (Liberalen) Intergouvernementalismus gleichzeitig die einzig legitime und relevante Form der Systemgestaltung repräsentiert. ${ }^{19}$ Die Stärke dieses Verfahrens liegt vor allem darin, dass nach der oftmals langwierigen Initiativphase und der nicht minder aufwendigen und politisch brisanten Verhandlungsphase davon auszugehen ist, dass die Ratifizierungsphase lediglich ein abschließender formaler Akt ist. Die getroffenen Beschlüsse stellen ein zwischen

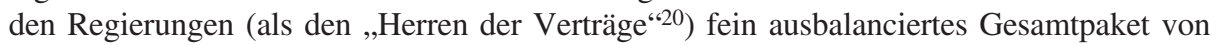
Kompromissen und ,package deals ‘ dar, das in der Ratifizierungsphase nur als Gesamtpaket anzunehmen oder abzulehnen ist. Da der europapolitische Schaden in letzterem Fall für die einzelnen Mitgliedstaaten kaum kalkulierbar ist, geht hiervon ein großer Ratifizierungsdruck insbesondere auf die nationalen Parlamente aus, auch wenn keinesfalls von einem Ratifizierungsautomatismus gesprochen werden kann - wie insbesondere die gescheiterten Referenden zu den Verträgen von Maastricht und Nizza belegen..$^{21}$

Im Umkehrschluss liegt die Schwäche des Verfahrens vor allem in der mangelnden Einbeziehung einer breiteren Öffentlichkeit und eines größeren Interessenspektrums sowie direkt gewählter Volksvertreter. Darüber hinaus waren die Ergebnisse von Regierungskonferenzen in den 1990er Jahren inhaltlich immer weniger überzeugend: Kompromisse wurden durch die Erhöhung der institutionellen und prozeduralen Komplexität erreicht, wie zum Beispiel die Regeln für qualifizierte Mehrheitsentscheidungen im Vertrag von Nizza belegen. Einige der lang umstrittenen Entscheidungen waren so weniger auf eine Stärkung der EU-Architektur ausgerichtet als vielmehr den Präferenzen der Mitgliedstaaten geschuldet. Der Vorwurf der Intransparenz, Ineffizienz und Bürgerferne führte im Anschluss an den Vertrag von Nizza zur „Erklärung von Laeken“22 und zur Einberufung des Verfassungskonventes, der Vorschläge für die weitere Ausgestaltung der Europäischen Union erarbeiten sollte.

\section{Die Konventsmethode}

Die Einführung der Konventsmethode stellt den Versuch dar, eine möglichst breite Palette von Interessen in den Prozess der konstitutionellen Weiterentwicklung der Europä-

19 Vgl. Andrew Moravcsik: What Can We Learn from the Collapse of the European Constitutional Project?, in: Politische Vierteljahresschrift 2/2006, S. 219-241; Andrew Moravcsik: Preferences and Power in the European Community: A Liberal Intergovernmentalist Approach, in: Journal of Common Market Studies, 30th Anniversary Edition, Dezember 1993.

20 Vgl. BVerfGE 89, 155, ,Maastricht‘.

21 Der Vertrag von Maastricht wurde im Referendum in Dänemark zuerst mit 50,7\% der Wähler im Juni 1992 abgelehnt, aber im zweiten Anlauf im Mai 1993 angenommen (56,7\%). Der Vertrag von Nizza wurde zuerst im Referendum in Irland mit 53,8\% der Wähler im Juni 2001 abgelehnt, aber ebenfalls im zweiten Anlauf angenommen $(62,89 \%)$.

22 Abrufbar unter: http://www.consilium.europa.eu/ueDocs/cms_Data/docs/pressData/de/ec/68829.pdf (letzter Zugriff: 21.09.07). 
ischen Union einzubeziehen, um dessen Ergebnissen eine tragfähigere Akzeptanz und damit auch eine größere Nachhaltigkeit zu garantieren. Dabei werden Akteure auf der europäischen (horizontalen) Ebene ebenso einbezogen wie Akteure auf der (vertikalen) nationalstaatlichen, regionalen und kommunalen Ebene. Idealtypisch und integrationstheoretisch ist die Konventsmethode damit dem Modell der „deliberativen Demokratie“ 23 zuzurechnen, das von der Überzeugungskraft guter Argumente und Lösungsvorschläge als Basis guten Regierens ausgeht. Die ,Macht' der beteiligten Akteure spielt in diesem Modell eine nachrangige Rolle, beziehungsweise speist sich weniger aus ihrer formalen Autorität, als vielmehr aus der Überzeugungskraft der von ihnen vertretenen Argumente.

Der im Dezember 2001 einberufene Verfassungskonvent war nach dem Grundrechtekonvent, der von Dezember 1999 bis Oktober 2000 unter der Leitung von Roman Herzog tagte, der zweite Versuch mit der Konventsmethode auf der europäischen Ebene. Der Verfassungskonvent war dabei zum ersten Mal mehrheitlich mit direkt gewählten Volksvertretern aus den nationalen Parlamenten und dem Europäischen Parlament besetzt, ${ }^{24}$ was eine unmittelbarere Rückbindung der Beratungen an die Interessen und Bedürfnisse der Bürgerinnen und Bürger in Europa gewährleisten sollte. In der Praxis der Konventsarbeit war jedoch insbesondere im Hinblick auf die Gestaltung der institutionellen Architektur - eine wesentlich größere Dominanz nationalstaatlicher Interessen- und Machtpolitik festzustellen, als idealtypisch anzunehmen und ursprünglich gehofft worden war. Stark kritisiert wurde dabei vor allem die oftmals eigenmächtige Vorgehensweise des Präsidiums des Konvents unter seinem Vorsitzenden Valéry Giscard d'Estaing. ${ }^{25}$ Der von den Mitgliedstaaten auf einer nachfolgenden Regierungskonferenz noch revidierte Verfassungsvertrag scheiterte jedoch in zwei Referenden. Die Gründe für das Scheitern des Vertrags in Frankreich und in den Niederlanden sind an anderer Stelle ausführlich analysiert worden. ${ }^{26}$ Es wäre jedoch sicherlich zu kurz gegriffen, aus der Ablehnung des Verfassungsvertrags grundsätzlich auf das Scheitern der Konventsmethode zu schließen.

Vielmehr bleiben als Stärken der Konventsmethode die erstmals so breit geführte Diskussion über die zukünftige Verfasstheit Europas hervorzuheben. Die vom Konvent erarbeiteten Ergebnisse bleiben in dem von der portugiesischen Präsidentschaft vorgelegten Entwurf des Reformvertrags in zentralen Fragen der institutionellen Architektur erhalten. ${ }^{27}$ Auch wenn die Konventsmethode vielen hochgesteckten Erwartungen im Hinblick auf den Anstoß zu einer umfassenden europaweiten, transnationalen Debatte über die Zukunft Europas nicht

23 Vgl. Christian Joerges/Jürgen Neyer: Von intergouvernementalem Bargaining zur deliberativen Politik: Gründe und Chancen für eine Konstitutionalisierung der europäischen Komitologie, in: Beate Kohler-Koch (Hrsg.): Regieren in entgrenzten Räumen, PVS-Sonderheft 28/1998, S. 207-233; Andreas Maurer: Die Methode des Konvents - Ein Modell deliberativer Demokratie?, in: integration 2/2003, S. 130-140; Daniel Göler: Deliberation - Ein Zukunftsmodell europäischer Entscheidungsfindung? Analyse der Beratungen des Verfassungskonvents 2002-2003, Baden-Baden 2006; Jürgen Habermas: Drei normative Modelle der Demokratie: Zum Begriff der deliberativen Demokratie, in: Jürgen Habermas: Die Einbeziehung des Anderen, Frankfurt a.M. 1996, S. 277-292.

24 Der Konvent setzte sich zusammen aus Repräsentanten von Exekutiven (Regierungen von 15 Mitgliedstaaten und 13 Beitrittskandidaten, zwei Vertreter der Kommission) und von Legislativen (jeweils zwei Abgeordnete der nationalen Parlamente und 16 des Europäischen Parlaments) sowie Beobachtern anderer Institutionen. Vom 28. Februar 2002 bis zum 10. Juli 2003 erarbeitete er, untergliedert in diverse Arbeitsgruppen, den Vertrag über eine Verfassung für Europa.

25 Peter Norman: The Accidental Constitution. The Story of the European Convention, Brüssel 2003.

26 Joachim Schild: Ein Sieg der Angst - das gescheiterte französische Verfassungsreferendum, in: integration 3/ 2005, S. 187-200; Mathias Jopp/Gesa S. Kuhle: Wege aus der Verfassungskrise - die EU nach den gescheiterten Referenden in Frankreich und den Niederlanden, in: integration 3/2005, S. 257-261.

27 Daniel Göler/Hartmut Marhold: Die Konventsmethode, in: integration 4/2003, S. 317-330; Elmar Brok: Der Konvent - eine Chance für die Europäische Verfassung, in: integration 4/2003, S. 338-344. 
vollständig gerecht geworden ist, so hat sie als Methode durchaus das Potenzial, auch zu zukünftigen Systemgestaltungsprozessen einen wichtigen Beitrag zu leisten. Dabei kann die Konventsmethode insbesondere dazu dienen, strittige Punkte vorab zu diskutieren und Lösungsvorschläge zu erarbeiten. Eine entsprechende Beteiligung des Konvents sieht der Entwurf des Reformvertrags auch beim „Ordentlichen Änderungsverfahren“ vor.

\section{Die ,Methode Monnet'}

Unter der ,Methode Monnet" wird eine pragmatische, schrittweise Vorgehensweise bei der Systemgestaltung der Europäischen Union verstanden, die konkrete Aufgaben für ein „Europa der Projekte“28 ins Zentrum stellt und - von Vorhaben in einzelnen Politikfeldern ausgehend - die institutionell und konstitutionell erforderlichen Rahmenbedingungen definiert und schafft (,form follows function“29). Die Systemgestaltung der europäischen polity entwickelt sich dabei in klassisch funktionalistischer Weise in erster Linie in den ,Tälern * zwischen großen Regierungskonferenzen und Vertragswerken, wenn die bestehenden Regelungen in die Praxis umgesetzt und erprobt werden. Folgerichtig spielen im Rahmen dieser Methode neben den nationalen Regierungen eine Reihe von weiteren Akteuren wie die Europäische Kommission oder der Europäische Gerichtshof eine wichtige Rolle im Systemgestaltungsprozess, obwohl sie bei Regierungskonferenzen nicht als gleichberechtigte Verhandlungspartner mitwirken..$^{30}$

Die undramatische und scheinbar unpolitische Art der Politikgestaltung und Systementwicklung, die von Jean Monnet und Robert Schuman in den 1950er Jahren geprägt wurde, ist in der integrationstheoretischen Debatte eng mit dem Neofunktionalismus verknüpft, der ebenfalls von einer schrittweisen, funktional definierten Ausweitung der (wirtschaftlichen) Zusammenarbeit ausgeht (,spill-over"), bis diese auch politische und konstitutionelle Fragen erfasst. ${ }^{31}$

Bei der ,Methode Monnet' spielte darüber hinaus auch die persönliche Strategie Monnets eine wichtige Rolle, die auf vertraulichen Kontakten und Absprachen mit den Entscheidungsträgern in Europa aufbaute, wobei ihm frühere Verdienste und seine persönliche Autorität zugute kamen. ${ }^{32}$ Für Monnet war die enge Zusammenarbeit zwischen Frankreich und Deutschland der Schlüssel zum Gelingen des europäischen Einigungsprojektes, das seiner Auffassung nach am ehesten durch eine ,Solidarität der Tat ${ }^{\text {“33 }}$ zu schaffen sein würde, das heißt durch ein konkretes, realistisches wirtschaftliches Projekt von politischer Relevanz, das deutsche wie französische Interessen berücksichtigte. Der institutionelle und konstitutionelle Rahmen für diese Politik war dabei zunächst Mittel zum Zweck, aber kein eigenständiges Projekt.

Die Stärke dieser Methode zeigt sich in der Integrationsgeschichte von den 1950er Jahren bis in die späten 1990er Jahre, die geprägt waren durch die schrittweise, evolutionäre Ausweitung der Kompetenzen der Gemeinschaft und die institutionelle Vertiefung der Zusammenarbeit in Abwesenheit einer klar definierten Finalität. Gerade die Mehrdeutigkeit bezie-

28 José Manuel Barroso: Rede vor dem Europäischen Parlament, 15./16. Juni 2006, abrufbar unter: http:// www.ec.europa.eu/commission_barroso/president/pdf/speech_20060614_de.pdf (letzter Zugriff: 21.09.07).

29 Vgl. David Mitrany: A Working Peace System, Oxford 1943.

30 Vgl. Thomas Christiansen/Knud Erik Jørgensen: Negotiating Treaty Reform in the European Union: The Role of the European Commission, in: International Negotiation 3/1998, S. 435-452, hier S. 437.

31 Vgl. Ernst B. Haas: The Uniting of Europe, Stanford 1958.

32 Vgl. Jean Monnet: Memoiren eines Europäers, München 1980; Wolfgang Wessels: Jean Monnet - Mensch und Methode. Überschätzt und überholt?, Reihe Politikwissenschaft des IHS, Wien, Band 74/2001.

33 Robert Schuman: Erklärung vom 9. Mai 1950 (Schuman-Erklärung), abrufbar unter: http://europa.eu/abc/symbols/9-may/decl_en.htm (letzter Zugriff: 21.09.07). 
hungsweise Offenheit der institutionellen Architektur wird von vielen Beobachtern als Garant für die Dynamik einer inkrementalistischen Systementwicklung zwischen den unterschiedlichen Interessen gewertet. ${ }^{34}$ Eine solche evolutionäre Systemgestaltung folgt dem Prinzip von „Versuch und Irrtum“ (,trial-and-error“), das für ein solch neuartiges und singuläres politisches System wie die Europäische Union angemessen erscheint. ${ }^{35}$

Die Schwächen der ,Methode Monnet', das heißt ihre Intransparenz, die extrem gesteigerte Komplexität der institutionellen Struktur der Europäischen Union und der (scheinbar) fehlende politische Ehrgeiz in einer Zeit, in der Europa dringend eines politischen Projektes bedurft hätte, wurden dagegen zum Ende der 1990er Jahre immer deutlicher sichtbar. ${ }^{36}$ So kündigte der damalige Außenminister Joschka Fischer in seiner weiterhin lesenwerten Humboldt-Rede im Jahr 2000 das Ende der „Methode Monnet“ zugunsten einer breiten politischen Debatte an, ${ }^{37}$ die im Dezember 2001 in die Einberufung eines Verfassungskonventes mündete.

\section{Die Entstehung der ,Road map“ unter deutscher Ratspräsidentschaft: Renaissance der ,Methode Monnet'}

Angesichts der Relevanz der Initiativphase von Regierungskonferenzen beim ,Agendasetting ' und bei ersten Orientierungen zu den geplanten Vertragsänderungen muss der Form und den Inhalten dieser Phase eine große Bedeutung im politischen Prozess und in der wissenschaftlichen Analyse eingeräumt werden. Speziell im Hinblick auf diese Initiativphase hat die deutsche Präsidentschaft in der ersten Jahreshälfte 2007 eine spezifische Variante entwickelt. Dabei hat sie in einer vollständigen Abkehr von der Konventsmethode auf die ,Methode Monnet' zurückgegriffen.

Im Anschluss an die Reflexionsphase nach den gescheiterten Referenden in Frankreich und den Niederlanden hatte der Europäische Rat die deutsche Präsidentschaft 2006 beauftragt, ,,in der ersten Jahreshälfte 2007 einen Bericht vor[zu]legen, der sich auf ausführliche Konsultationen mit den Mitgliedstaaten stützt. Dieser Bericht sollte eine Bewertung des Stands der Beratungen über den Verfassungsvertrag enthalten und mögliche künftige Entwicklungen aufzeigen. " 38 Aufgrund dieses Mandats hat die Bundesregierung in mehreren Stufen sorgfältig und detailliert in einem intensiven Prozess bilateraler Gespräche und Absprachen mit einigen gemeinsamen Sitzungen mehrerer Mitgliedstaaten einen Text vorbereitet, der dann auf dem Abschlussgipfel teilweise sehr kontrovers verhandelt wurde, bevor er endgültig verabschiedet werden konnte. ${ }^{39}$

34 Vgl. zur Diskussion des Verfassungsvertrags Wolfgang Wessels: Die Europapolitik in der wissenschaftlichen Debatte, in: Werner Weidenfeld/Wolfgang Wessels (Hrsg.): Jahrbuch der Europäischen Integration 2006, Baden-Baden 2007, S. 27-38 insbesondere S. 30.

35 Eine „experimentelle Union“ ist demnach nur in einzelnen Schritte zu (er-)finden, und nicht durch einen konstitutionellen Akt ex nihilo zu schaffen. Vgl. Brigid Laffan/Imogen Sudbery: Zur Ratifizierungskrise des Verfassungsvertrages - drei politikwissenschaftliche Lesarten und ihre Kritik, in: integration 4/2006, S. 271-286.

36 Bereits in den 1960er Jahren hatte Stanley Hoffmann die „Seelenlosigkeit” der Methode Monnet kritisiert: „In order to go "beyond the nation-state", one will have to do more than set up procedures in adequate "background" and "process conditions'. For a procedure is not a purpose, a process is not a policy". Stanley Hoffmann: Obstinate or Obsolete? The Fate of the Nation State and the Case of Western Europe, in: Daedalus, Journal of the American Academy of Arts and Sciences 3/1966, S. 862-915, hier S. 881.

37 Vgl. Joschka Fischer: Vom Staatenverbund zur Föderation, 2000.

38 Europäischer Rat (Brüssel): Schlussfolgerungen des Vorsitzes, 15./16. Juni 2006, Ziffer 47, abrufbar unter: http://consilium.europa.eu/ueDocs/cms_Data/docs/pressData/de/ec/90120.pdf (letzter Zugriff 16.08.2007).

39 Vgl. Andreas Maurer: Vom Verfassungs- zum Reformvertrag, SWP Diskussionspapier Juli 2007, S. 136. 
Dem Idealtyp der Konventsmethode diametral entgegengesetzt wurde so ein „,vertraulicher Konsultationsprozess" $" 40$ hinter verschlossenen Türen durchgeführt, in dessen Verlauf nicht nur ein Fahrplan, sondern auch konkret formulierte Vorgaben für die weiteren Verhandlungen auf der Regierungskonferenz vereinbart wurden. ${ }^{41}$ Die deutsche Präsidentschaft hat so mit der Unterstützung des Ratssekretariats in kurzer Zeit den Weg für die Vertragsreform geebnet, wobei Ergebnisse des Verfassungskonvents und der Regierungskonferenz 2004 eine wesentliche Basis der Verhandlungen bildeten. Die Kunst beziehungsweise der Erfolg der deutschen Ratspräsidentschaft bestand jedoch darin, einen Konsens zu den besonders umstrittenen Regeln zur institutionellen Architektur zu erreichen. ${ }^{42}$ Dabei bediente sie sich einer Kombination verschiedener Techniken und Mittel:

- Einen umfangreichen Teil der Ergebnisse der Regierungskonferenz von 2004 erklärte die Präsidentschaft mit einer ,roten Linie' für ,sakrosankt'; dieser Bestand an abgesegneten Übereinkünften, der ,acquis‘ der vorangegangenen Regierungskonferenz, wurde nicht mehr in Frage gestellt. ${ }^{43}$

- Einige umstrittene Formulierungen, die der Europäischen Union verfassungs- beziehungsweise staatsähnliche Züge verliehen hätten, wurden gestrichen. Hierfür hatte die Berliner Erklärung zum 50-jährigen Gründungstag der Römischen Verträge den Weg vorgezeichnet. ${ }^{44}$

- Einige Streitpunkte wurden durch Ausnahmeregelungen insbesondere in Form von Optouts für das Vereinigte Königreich ausgegliedert.

- Andere Streitpunkte wie insbesondere die Neuregelung für Mehrheitsabstimmungen im Ministerrat wurden durch die Einführung einer komplizierten Fristenregelung mit weiteren Sonderregelungen einem Kompromiss zugeführt.

Zusätzlich zu diesen Punkten sind weitere spezifische Taktiken zu beobachten, die üblicherweise in Verhandlungen im europäischen und anderen politischen Systemen angewendet werden. ${ }^{45}$ So wurde mit der Benennung eines konkreten Termins für das Inkrafttreten des Reformvertrags (vor den Wahlen zum Europäischen Parlament 2009) ein mittelfristiger Zeitdruck aufgebaut. Von diesem Termin ausgehend wurde den Verhandlungspartnern vermittelt, dass der Gipfel als letzte Chance für einen Durchbruch zu verstehen sei. Darüber hinaus mobilisierte die deutsche Ratspräsidentschaft vielfältige Kontakte und Einflusskanäle auf mehreren Ebenen. Berichtet wurde von Verhandlungen in kleinen Gruppen und auch von

40 Thomas de Maizière: Die deutsche EU-Ratspräsidentschaft - eine Bilanz, Rede an der Rechtswissenschaftlichen Fakultät der Westfälischen Wilhelms-Universität in Münster am 28.6.07, abrufbar unter: http://bundesregierung.de/nn_1498/Content/DE/Rede/2007/06/2007-06-28-rede-chefbk-uni-muenster.html (letzter Zugriff: 21.09.07).

41 Vgl. Andreas Maurer: Rückbau, Raubbau, Wiederaufbau. Die Arbeiten zum Verfassungsvertrag, in: Daniela Kietz/Volker Perthes (Hrsg.): Handlungsspielräume einer EU-Ratspräsidentschaft. Eine Funktionenanalyse des deutschen Vorsitzes im ersten Halbjahr 2007, SWP-Studie 2007/S 24, September 2007, S. 27-33.

$42 \mathrm{Zu}$ nennen sind hier unter anderem die Mehrheitsregeln im Rat, die Grundrechtecharta, der Gottesbezug und das Amt des ,Europäischen Außenministers‘.

43 Hiervon zeugen die zahlreichen Verweise des Mandats auf unverändert zu übernehmende Ergebnisse der Regierungskonferenz 2004, vgl. exemplarisch zum Verfahren über die verstärkte Zusammenarbeit: „Der Titel IV (früher Titel VII des bestehenden EUV) wird wie auf der RK 2004 vereinbart geändert." Europäischer Rat: Mandat für die Regierungskonferenz 2007, Ziffer 14.

44 Vgl. Timo Goosmann: Die ,Berliner Erklärung - Dokument europäischer Identität oder pragmatischer Zwischenschritt zum Reformvertrag?, in: integration 3/2007, S. 251-263; Die Berliner Erklärung ist abrufbar unter: http:// www.eu2007.de/de/About_the_EU/Constitutional_Treaty/BerlinerErklaerung.html (letzter Zugriff: 21.09.2007); Daniela Schwarzer: Die Berliner Erklärung - Testlauf für die Verhandlungen zum Verfassungsvertrag, in: Daniela Kietz/Volker Perthes (Hrsg.): Handlungsspielräume einer EU-Ratspräsidentschaft, 2007, S. 20-26.

45 Vgl. zu Regierungskonferenzen insbesondere Thomas Christiansen/Gerda Falkner/Knud E. Jørgensen: Theorising EU Treaty Reform: Beyond Diplomacy and Bargaining, in: Journal of European Public Policy 1/2002, S. 12-32. 
Bemühungen, außerhalb des Ratsgebäudes auf einzelne Teilnehmer einzuwirken. Über den Durchbruch am frühen Morgen des 23. Juni 2007 gaben Beteiligte unterschiedliche Berichte ab und nahmen abweichende Erfolgszuweisungen vor, wobei der deutschen Präsidentschaft und insbesondere der Bundeskanzlerin jedoch besondere Anerkennung ausgesprochen wurde. ${ }^{46}$

$\mathrm{Zu}$ diskutieren ist, ob und in welchem Umfang die Vorgehensweise der deutschen Ratspräsidentschaft mit zentralen Charakteristika der ,Methode Monnet' verglichen werden kann. Einige Merkmale ähneln sich deutlich: So entwickelte die Bundesregierung eine auf die einzelnen Akteure abgestimmte Strategie und propagierte eine vertrauliche Arbeit hinter den Kulissen. Diese Vorgehensweise zielte darauf ab, das Vertrauen der relevanten Akteure zu gewinnen. Durch eine umfassende und frühzeitige Einbindung in die Ausgestaltung des Mandats der deutschen Ratspräsidentschaft verpflichteten sich alle Beteiligten gleichzeitig zunehmend auf Prozeduren und Substanz des neuen Textes. Dabei wurde besonderer Nachdruck auf für alle Beteiligte transparente und nachvollziehbare Verfahren gelegt, während die Öffentlichkeit mit der Berliner Erklärung auf die Grundrichtung des neuen Vertragstextes eingestimmt wurde. Dieser sach- und lösungsorientierte Verhandlungsstil und die vertraulich geführten Gespräche erleichterten die Konsensfindung und ebneten den Staats- und Regierungschefs den Weg zu Kompromisslösungen, ohne in der nationalen Öffentlichkeit einen Gesichtsverlust riskieren zu müssen. Dem Prozess kam zugute, dass Merkel - wie in den ersten Jahrzehnten der Integrationskonstruktion Monnet - nicht zuletzt aufgrund ihres Lebenswegs eine persönliche Autorität zugeschrieben wurde, die für die direkten Verhandlungen einen Sympathiebonus bedeutete. Wie Monnet präsentierte Merkel dabei ein glaubwürdiges Bekenntnis zu einer nicht eindeutig festgelegten Zukunft der Integrationskonstruktion, deren Legitimität auf grundlegenden Werten beruht, aber gleichzeitig spezifische nationale Interessen miteinander in Einklang bringen soll, und - wie Monnet - setzte die Bundesregierung anstelle einer umfassenden Konstitutionalisierungsstrategie auf eine Politik der kleinen, aber realisierbaren Schritte. Dabei musste auch die Bundeskanzlerin höhergesteckte Erwartungen enttäuschen, um einen Stillstand zu verhindern. Schließlich war für Merkel wie für Monnet die deutsch-französische Verständigung die notwendige Voraussetzung für das Gelingen der Reformbemühungen.

Zugleich lässt sich ein deutlicher Unterschied festhalten: Anders als bei der klassischen ,Methode Monnet` hatte für Merkel nicht ein einzelnes Vorhaben im Sinne eines ,Europa der Projekte', sondern die Neustrukturierung der Aufgaben- und Zuständigkeitsverteilung und die Ausgestaltung der institutionellen Architektur oberste Priorität. Zur Energie- und Klimapolitik, die sich als ,Lokomotiven“ angeboten hätten, sah das Mandat zwar begrenzt weitergehende Formulierungen für den Reformvertrag vor; diesen kam aber im Verhandlungsprozess nicht die zentrale Bedeutung zu, wie sie zum Beispiel die Währungsunion im Maastrichter Vertrag eingenommen hatte. Die Beschlüsse des Märzgipfels 2007 zum Aktionsplan „Eine Energiepolitik für Europa“ ${ }^{47}$ können als Baustein in dem Prozess verstanden werden, der zum Juni-Rat führte, aber im Unterschied zu den Römischen Verträgen und zum Unionsvertrag von Maastricht ging keine Dynamik von einem Schlüsselbereich europäischer Politik aus. Stattdessen dominierten institutionelle Aspekte den Gipfel und die Verhandlungen im Rahmen der neuerlichen Regierungskonferenz.

46 Vgl. Nicolas Sarkozy: Rede zur Eröffnung der 15. Botschafterkonferenz, 27.08.07 in Paris, abrufbar unter: http://www.elysee.fr/elysee/elysee.fr/francais/interventions/2007/aout/allocution_a_1_occasion_de_la_conferen ce_des_ambassadeurs.79272.html (letzter Zugriff: 21.09.2007).

47 Vgl. Europäischer Rat (Brüssel): Schlussfolgerungen des Vorsitzes, 8./9. März 2007, abrufbar unter: http:// www.consilium.europa.eu/ueDocs/cms_Data/docs/pressData/de/ec/93139.pdf (letzter Zugriff: 01.10.2007). 
Diese Differenz zur ursprünglichen ,Methode Monnet' lässt sich in erster Linie durch den Auftrag an die deutsche Ratspräsidentschaft erklären, der sich explizit auf die Neuordnung der institutionellen Architektur und die Aufnahme der Grundrechtecharta in den Vertrag bezog. Darüber hinaus erklärt sich diese unterschiedliche Schwerpunktsetzung auch durch den erreichten Integrationsstand, der die Klärung grundlegender institutioneller und konstitutioneller Fragen dringlicher erscheinen ließ als eine erneute Erschließung weiterer zentraler Politikfelder. Es bleibt jedoch offen, ob es der deutschen Ratspräsidentschaft mit dieser Vorgehensweise tatsächlich gelungen ist, ein robustes Verhandlungspaket zu schnüren, das für jeden der teilnehmenden Staats- und Regierungschefs eine ausreichende Basis für die innenpolitische Durchsetzung bietet. Dabei ist insbesondere fraglich, wie die Debatten während der Ratifizierungsphase des Reformvertrags verlaufen werden, wenn den Bürgerinnen und Bürgern kein ehrgeizigeres beziehungsweise für sie unmittelbar relevantes Projekt angeboten wird. Wie schon beim Verfassungsvertrag ${ }^{48}$ wird auch die Ratifizierung des Reformvertrags dadurch erschwert werden, dass seine Inhalte schwerer vermittelbar sind, da sie außerhalb der Erfahrungswelt der Unionsbürgerinnen und -bürger liegen: So sind die Auswirkungen einer einheitlichen Währung oder einer gemeinsamen europäischen Verteidigungspolitik wesentlich klarer zu diskutieren und einfacher zu vermitteln als die potenziellen Effekte einer veränderten Mehrheitsregelung im Ministerrat.

Aus integrationstheoretischer Perspektive erhöht diese Entkoppelung von konstitutioneller Weiterentwicklung und politikfeldspezifischer Vertiefung die Schwierigkeit, ein ausgewogenes Verhandlungspaket mit Gewinnen für jeden Verhandlungspartner zu erreichen. ${ }^{49}$ Gerade die enge Koppelung von institutionellen Reformen und inhaltlicher Vertiefung war es, die nach Ansicht vieler Beobachter bei früheren Vertragsverhandlungen den entscheidenden Durchbruch zwischen den Verhandlungspartnern ermöglichte: ,[...] [I]nstitutional reform is easier to achieve when it is not pursued for its own sake, but emerges as a logical implication of substantive policy choices." ${ }^{50}$ Im Umkehrschluss führt eine Entkoppelung von institutionellen und politischen Projekten dazu, dass keine policy-basierte Kompensation für eine weitere institutionelle und konstitutionelle Ausgestaltung des EU-Systems (und damit eines weitergehenden nationalen Souveränitätsverlustes) angeboten werden kann, was die Ratifizierung institutioneller Reformprojekte durch nationale Parlamente und Referenden deutlich erschwert, wenn nicht gar unmöglich macht.

\section{Schlussfolgerungen: Vom Verfassungskonvent zum Methodenmix}

Die Suche nach einer Finalität der Integrationskonstruktion ist in eine neue Phase eingetreten, die aber erneut nicht das Ende der Selbstverständigung über das politische System der Europäischen Union markieren dürfte. Auch wenn das „Verfassungskonzept“ vom Europäischen Rat aufgegeben wurde, ${ }^{51}$ so können die Staats- und Regierungschefs damit eine weitergehende Debatte um die Finalität der Integrationskonstruktion auf Dauer nicht unterbinden: ,[D]ie Funktion des Verfassungstopos“ erweist sich als ,zeitlos auch im europäischen

48 Hinsichtlich dieser Problematik in Bezug auf den Verfassungsvertrag vgl. Udo Diedrichs/Wolfgang Wessels: Die Europäische Union in der Verfassungsfalle? Analysen, Entwicklungen und Optionen, in: integration 4/ 2005, S. 287-306.

49 Vgl. Anne Faber/Wolfgang Wessels: Strategien und institutionelle Perspektiven nach der Verfassungskrise: ,Funktionalistische' und ,institutionalistische' Wege zu einem neuen europäischen Verhandlungspaket, in: Politische Vierteljahresschrift 2/2006, S. 252-263.

50 Vgl. stellvertretend für viele Renaud Dehousse/Giandomenico Majone: The Institutional Dynamics of European Integration: From the Single Act to the Maastricht Treaty, in: Stephen Martin (Hrsg.): The Construction of Europe. Essays in Honour of Emile Noël, Dordrecht 1994, S. 91-112, hier S. 104.

51 Europäischer Rat: Mandat für die Regierungskonferenz 2007, Ziffer 1. 
Kontext“ ${ }^{52}$ Der ,,constitutional turn“"53 in der europäischen Politik und in der Integrationswissenschaft wird sich nicht einfach als überholt ablegen lassen. ${ }^{54}$

Gelingt die Verabschiedung des Reformvertrags in diesem Jahr und die Ratifizierung im Jahre 2008, so ist dieser Prozess als Ergebnis des Zusammenspiels von Konventsmethode, zweier Regierungskonferenzen mit dem Europäischen Rat als „konstitutionellem Architekten " 55 und der von Merkel eingeleiteten Renaissance der ,Methode Monnet" des sachorientierten Verhandlungsstils und der vertraulichen Gespräche zu verstehen. Dabei rahmen die Regierungskonferenzen von 2004 und 2007 die Neugestaltung der europäischen Grundlagenverträge in zwei klassische intergouvernementale Verhandlungsprozesse ein, in denen die Mitgliedstaaten erneut hartnäckig um einzelne Aspekte der Systemgestaltung gerungen haben und weiter ringen. Die besondere Schwierigkeit bei der Unterzeichnung und Ratifizierung des Reformvertrags liegt dabei wie schon beim Vertrag von Nizza und insbesondere beim Verfassungsvertrag in der Entkoppelung institutioneller Reformen von der Weiterentwicklung gemeinsamer Politiken.

Die Konventsmethode, mit dem Verfassungskonvent zum zweiten Mal angewandt, hat als neueste Methode eine noch genauer zu analysierende Rolle bei der Neugestaltung der vertraglichen Grundlagen der Europäischen Union gespielt. Diese bleibt von Interesse, auch wenn die Arbeit letztlich nicht wie vom Konvent angestrebt in eine Verfassung für Europa mündete, sondern zentrale Elemente des Reformvertrags vorzeichnete. Dennoch ist dieses Verfahren nicht obsolet, sondern wird - entschlackt um hochgesteckte Erwartungen - zukünftig weiterhin eine wichtige ergänzende Rolle bei der , deliberativen' Vorbereitung von Vertragsänderungen spielen. Vorteil und Mehrwert der Konventsmethode bleibt, dass sie eine breitere argumentative Basis für Reformen und Veränderungen schaffen kann als dies Regierungskonferenzen und die ,Methode Monnet' allein leisten könnten.

Entgegen der Ankündigung von Joschka Fischer im Jahr 2000 ist die ,Methode Monnet“ nicht überholt, sondern wurde von der deutschen Präsidentschaft in Form von sach- und lösungsorientierten, vertraulichen Gesprächen wieder belebt. Als Politikerin und Regierungschefin nutzte die Bundeskanzlerin allerdings auch die im Europäischen Rat üblichen härteren Verhandlungsinstrumente wie Drohkulissen. Auch wenn sich der Erfolg der ,Methode Merkel' frühestens Ende 2008 nach dem Abschluss der Ratifizierung des Reformvertrags verlässlich abschätzen lassen wird, so ist das Vorgehen der Bundesregierung als ein weiteres Lehrstïck in die Liste der Verfahren und Strategien bei der Integrationskonstruktion aufzunehmen.

52 Peter-Christian Müller-Graff: Die Zukunft des europäischen Verfassungstopos, in: integration 3/2007, S. 223237, hier S. 236.

53 Thomas Diez/Antje Wiener: Introducing the Mosaic of Integration Theory, in: Thomas Diez/Antje Wiener (Hrsg.): Theories of European Integration: Past, Present and Future, Oxford 2004, S. 1-21, hier S. 10.

54 Vgl. Anne Faber: Die Weiterentwicklung der Europäischen Union: Vertiefung versus Erweiterung?, in: integration $2 / 2007$, S. 103-116, hier S. 115.

55 Wolfgang Wessels: Das politische System der Europäischen Union, Wiesbaden (im Erscheinen). 